\title{
Contact characteristics of beveloid gears
}

\author{
Chia-Chang Liu, Chung-Biau Tsay * \\ Department of Mechanical Engineering, National Chiao Tung University, Hsinchu, 30010, Taiwan \\ Received 19 October 2000; accepted 4 November 2001
}

\begin{abstract}
This study investigates the contact characteristics of beveloid gear pairs with intersected, crossed and parallel axes. Tooth contact analysis (TCA) is performed to examine the meshing and bearing contact of the beveloid gear pairs composed of a pinion and a gear. In addition, the principal directions and curvatures of the pinion and gear surfaces are investigated and the contact ellipses of the mating tooth surfaces are also studied. Numerical illustrative examples are provided to demonstrate the computational results. (C) 2002 Elsevier Science Ltd. All rights reserved.
\end{abstract}

\section{Introduction}

Beveloid gears, also known as conical involute gears, are involute gears with tapered tooth thickness, root and outside diameters. This type of gears can transmit rotational motion between intersected, crossed and parallel axes in any relative position. Owing to its tapered tooth thickness, the beveloid gear can also be used as the backlash control gears. Moreover, under non-parallel axes meshing, beveloid gears are not sensitive to assembly errors. The meshing of beveloid gears has seldom been studied, and Mitome [1-4] has conducted most of the researches in this area. The tooth action of a beveloid gear pair was investigated both theoretically and experimentally by Mitome [1]. His investigation verified that beveloid gear pairs transmit a uniform rotational motion between intersected axes even when assembly errors exist. Mitome [2] also proposed a design for the crossed axes beveloid gears and established their engagement models. In addition, the concept of infeed grinding which can enlarge the contact patterns of straight beveloid gears with intersected axes was proposed [3]. More recently, Mitome [4] also proposed a novel design for miter beveloid gears, which enables the tooth bearing contact located at the middle region of the tooth width.

\footnotetext{
${ }^{*}$ Corresponding author. Tel.: +886-3-5728450; fax: +886-3-5728450.

E-mail address: cbtsay@cc.nctu.edu.tw (C.-B. Tsay).
} 
Although the above investigations contributed significantly to the meshing of beveloid gears, they failed to establish a complete simulation model for tooth contact analysis (TCA). The TCA method was proposed by Litvin [5,6], and it has been applied to simulate the meshing of various types of gear drives. The TCA results provide valuable information on the contact points, contact lines, lines of action and transmission error (TE) of the mating gear pair during their meshing process. This study investigates the meshing characteristics of beveloid gear pairs with intersected, crossed and parallel axes. Theoretically, the bearing contact of a beveloid gear pair under nonparallel axes meshing is a point contact. Due to the elasticity, the gear tooth contact is spread over an elliptical area around the instantaneous contact point. The direction and dimension of the contact ellipse provide further contact and lubrication characteristics to the gear meshing. In this work, methodologies proposed by Litvin $[5,6]$ considering the principal directions and curvatures of mating surfaces was adopted to obtain the contact ellipses of the non-parallel axes beveloid gear pairs. The numerical illustrative examples accurately reflect the contact nature of a beveloid gear pair under several assembly conditions.

\section{Mathematical model of beveloid gear pairs}

According to Merritt's generation concept [7], a beveloid gear can be generated by a basic rack whose pitch plane intersects with the axis of the gear, and forms an angle that equals the generating cone angle. In industrial applications, the most conventional means of beveloid gear manufacturing method is the taper hobbing proposed by Mitome [8,9]. According to Mitome's results, the envelope generated by the hob in the space can be considered a rack cutter. In this work, the rack cutter is used to simulate the generating process of beveloid gears.

\subsection{Mathematical model of rack cutter}

The beveloid gear pair for the meshing simulation comprises a pinion and a gear. Assume that the cutter surface $\Sigma_{F}$ generates the pinion tooth surface $\Sigma_{1}$, and the cutter surface $\Sigma_{G}$ generates the gear tooth surface $\Sigma_{2}$. Notably, subscripts $i=1$ and 2 , and $j=F$ and $G$ represent the surfaces of pinion $\Sigma_{1}$ and gear $\Sigma_{2}$ and their corresponding cutters $\Sigma_{F}$ and $\Sigma_{G}$, respectively, in the following derivation. According to Fig. 1, the normal section of the rack cutter consists mainly of two

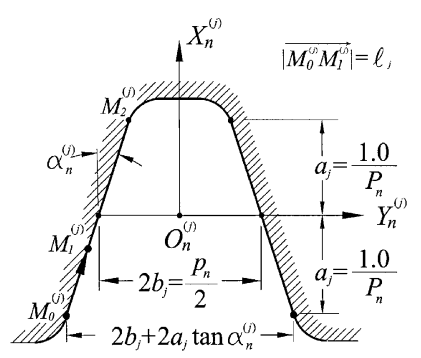

Fig. 1. The normal section of rack cutter. 
straight edges. The straight edge $\overline{M_{0}^{(j)} M_{2}^{(j)}}$ can be represented in coordinate system $S_{n}^{(j)}\left(X_{n}^{(j)}, Y_{n}^{(j)}, Z_{n}^{(j)}\right)$ by

$$
\begin{aligned}
& x_{n}^{(j)}=\ell_{j} \cos \alpha_{n}^{(j)}-a_{j}, \\
& y_{n}^{(j)}=\ell_{j} \sin \alpha_{n}^{(j)}-a_{j} \tan \alpha_{n}^{(j)}-b_{j}, \\
& z_{n}^{(j)}=0,
\end{aligned}
$$

where design parameter $\ell_{j}=\left|\overrightarrow{M_{0}^{(j)} M_{1}^{(j)}}\right|$ represents the distance measured from the initial point $M_{0}^{(j)}$, moving along the straight line $\overline{M_{0}^{(j)} M_{2}^{(j)}}$, to point $M_{1}^{(j)} ; \alpha_{n}^{(j)}$ denotes the normal pressure angle, and symbols $P_{n}$ and $p_{n}$ represent the gear diametral pitch and circular pitch, respectively.

To obtain the rack cutter surface for beveloid gear generation, the above-mentioned normal section of the rack cutter, attached to plane $X_{n}^{(j)}-Y_{n}^{(j)}$, is translated along the line $\overline{O_{r}^{(j)} O_{n}^{(j)}}$ with respect to the coordinate system $S_{r}^{(j)}\left(X_{r}^{(j)}, Y_{r}^{(j)}, Z_{r}^{(j)}\right)$, as illustrated in Fig. 2. Herein, $u_{j}=\left|\overrightarrow{O_{r}^{(j)} O_{n}^{(j)}}\right|$ is also a design parameter of the rack cutter surface. Thus, the profile of the rack cutter can be traced out in coordinate system $S_{r}^{(j)}$, and plane $Y_{r}^{(j)}-Z_{r}^{(j)}$ can be regarded as the pitch plane of the rack cutter. The angle $\beta_{j}$, which determines the direction of tooth trace, is the helix angle of tooth trace on the pitch plane of rack cutter. To simulate the taper hobbing process, the pitch plane of the rack cutter is set to have an inclination angle $\delta_{i}$ with respect to the plane axode coordinate system $S_{c}^{(j)}\left(X_{c}^{(j)}, Y_{c}^{(j)}, Z_{c}^{(j)}\right)$, and the rack cutter surface can be represented in coordinate system $S_{c}^{(j)}$ as follows:

$$
\begin{aligned}
& x_{c}^{(j)}=\left(\ell_{j} \cos \alpha_{n}^{(j)}-a_{j}\right) \cos \delta_{i}+\left[-\left(\ell_{j} \sin \alpha_{n}^{(j)}-a_{j} \tan \alpha_{n}^{(j)}-b_{j}\right) \sin \beta_{j}+u_{j} \cos \beta_{j}\right] \sin \delta_{i}, \\
& y_{c}^{(j)}=\left(\ell_{j} \sin \alpha_{n}^{(j)}-a_{j} \tan \alpha_{n}^{(j)}-b_{j}\right) \cos \beta_{j}+u_{j} \sin \beta_{j}, \\
& z_{c}^{(j)}=-\left(\ell_{j} \cos \alpha_{n}^{(j)}-a_{j}\right) \sin \delta_{i}+\left[-\left(\ell_{j} \sin \alpha_{n}^{(j)}-a_{j} \tan \alpha_{n}^{(j)}-b_{j}\right) \sin \beta_{j}+u_{j} \cos \beta_{j}\right] \cos \delta_{i} .
\end{aligned}
$$

Owing to that the surface coordinates of rack cutter are $\ell_{j}$ and $u_{j}$, the unit normal to the rack cutter surface can be attained by

$$
\boldsymbol{n}_{c}^{(j)}=\frac{\boldsymbol{N}_{c}^{(j)}}{\left|\boldsymbol{N}_{c}^{(j)}\right|} \quad(j=F, G),
$$

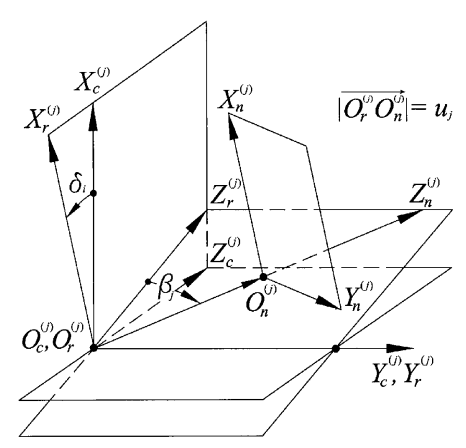

Fig. 2. Relations among coordinate systems $S_{n}^{(j)}, S_{r}^{(j)}, S_{c}^{(j)}$. 
where

$$
\boldsymbol{N}_{c}^{(j)}=\frac{\partial \boldsymbol{R}_{c}^{(j)}}{\partial u_{j}} \times \frac{\partial \boldsymbol{R}_{c}^{(j)}}{\partial \ell_{j}} .
$$

Herein, $\boldsymbol{R}_{c}^{(j)}$ denotes the position vector of the rack cutter surface represented in plane axode coordinate system $S_{c}^{(j)}$. Eqs. (2) and (3) result in the corresponding unit normals to the rack cutter surface as follows:

$$
\begin{aligned}
n_{x c}^{(j)} & =-\sin \delta_{i} \sin \beta_{j} \cos \alpha_{n}^{(j)}-\cos \delta_{i} \sin \alpha_{n}^{(j)}, \\
n_{y c}^{(j)} & =\cos \beta_{j} \cos \alpha_{n}^{(j)}, \\
n_{z c}^{(j)} & =-\cos \delta_{i} \sin \beta_{j} \cos \alpha_{n}^{(j)}+\sin \delta_{i} \sin \alpha_{n}^{(j)} .
\end{aligned}
$$

\subsection{Mathematical model of beveloid gear}

Fig. 3 illustrates a schematic generation mechanism and the coordinate relationship between the rack cutter and the generated gear. Herein, $S_{b}^{(j)}\left(X_{b}^{(j)}, Y_{b}^{(j)}, Z_{b}^{(j)}\right)$ is the fixed coordinate system, $S_{i}\left(X_{i}, Y_{i}, Z_{i}\right)$ is the coordinate system attached to the generated pinion $\Sigma_{1}(i=1)$ or gear $\Sigma_{2}(i=2)$, and $S_{c}^{(j)}$ is the plane axode coordinate system attached to the rack cutter. In the gear generation process, the gear blank rotates with angular velocity $\omega_{i}$ while the rack cutter translates with velocity $\boldsymbol{V}=\omega_{i} r_{i}$. The plane axode and the gear axode roll over each other without sliding on the instantaneous axis of rotation I-I. Based on the theory of gearing [5,6], the mathematical model of the generated beveloid gear tooth surfaces can be attained in coordinate system $S_{i}$ as follows:

$$
\begin{aligned}
x_{i} & =x_{c}^{(j)} \cos \phi_{i}-y_{c}^{(j)} \sin \phi_{i}+r_{i}\left(\cos \phi_{i}+\phi_{i} \sin \phi_{i}\right), \\
y_{i} & =x_{c}^{(j)} \sin \phi_{i}+y_{c}^{(j)} \cos \phi_{i}+r_{i}\left(\sin \phi_{i}-\phi_{i} \cos \phi_{i}\right), \\
z_{i} & =z_{c}^{(j)}
\end{aligned}
$$

where

$$
\phi_{i}=\left(y_{c}^{(j)} n_{x c}^{(j)}-x_{c}^{(j)} n_{y c}^{(j)}\right) /\left(r_{i} n_{x c}^{(j)}\right)
$$

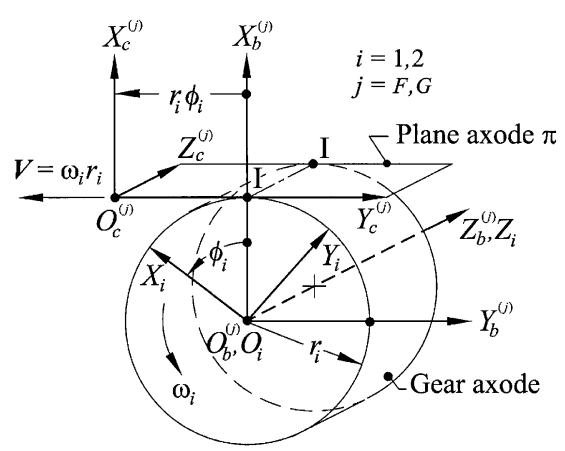

Fig. 3. Coordinate relationship between the rack cutter and generated gear. 
Herein, $i=1$ and 2 , and $j=F$ and $G$ represent the surfaces of pinion $\Sigma_{1}$ and gear $\Sigma_{2}$ and their corresponding cutters $\Sigma_{F}$ and $\Sigma_{G}$, respectively; $r_{i}$ denotes the pitch radius of the generated gear and $\phi_{i}$ represents the gear rotation angle in the generating process. Eq. (7) is the general form of the equation of meshing which can be obtained by applying the concept that the normal vector to any point on the generated tooth surface passes through the instantaneous axis of gear rotation II [5,6]. Substituting Eqs. (2) and (5) into Eqs. (6) and (7) yields the mathematical models of beveloid pinion $\Sigma_{1}(i=1$ and $j=F)$ and gear $\Sigma_{2}(i=2$ and $j=G)$ expressed in coordinate systems $S_{1}\left(X_{1}, Y_{1}, Z_{1}\right)$ and $S_{2}\left(X_{2}, Y_{2}, Z_{2}\right)$, respectively. The surface unit normal of the generated beveloid gear tooth surfaces can also be obtained by

$$
\begin{aligned}
& n_{i x}=n_{x c}^{(j)} \cos \phi_{i}-n_{y c}^{(j)} \sin \phi_{i}, \\
& n_{i y}=n_{x c}^{(j)} \sin \phi_{i}+n_{y c}^{(j)} \cos \phi_{i}, \\
& n_{i z}=n_{z c}^{(j)} .
\end{aligned}
$$

\section{Meshing model and tooth contact analysis}

Adopting the engagement model proposed by Mitome [2], Fig. 4 illustrates the schematic meshing model of the beveloid gear pair, including the pinion $\Sigma_{1}$, the gear $\Sigma_{2}$, and the pitch plane of the imaginary engaging rack. Beveloid pinion and gear can be considered two imaginary cones with cone angles $\delta_{1}$ and $\delta_{2}$, lying on opposite sides of the pitch plane of the imaginary engaging rack, while $S_{f}\left(X_{f}, Y_{f}, Z_{f}\right)$ and $S_{g}\left(X_{g}, Y_{g}, Z_{g}\right)$ are the reference coordinate systems for the pinion coordinate system $S_{1}\left(X_{1}, Y_{1}, Z_{1}\right)$ and gear coordinate system $S_{2}\left(X_{2}, Y_{2}, Z_{2}\right)$, respectively. Furthermore, $\phi_{1}^{\prime}$ and $\phi_{2}^{\prime}$ denote the rotation angles of the pinion and gear during meshing. Meanwhile, points $O_{f}$ and $O_{g}$ are the centers of the pitch circles of the pinion and gear, where $r_{1}$ and $r_{2}$ denote

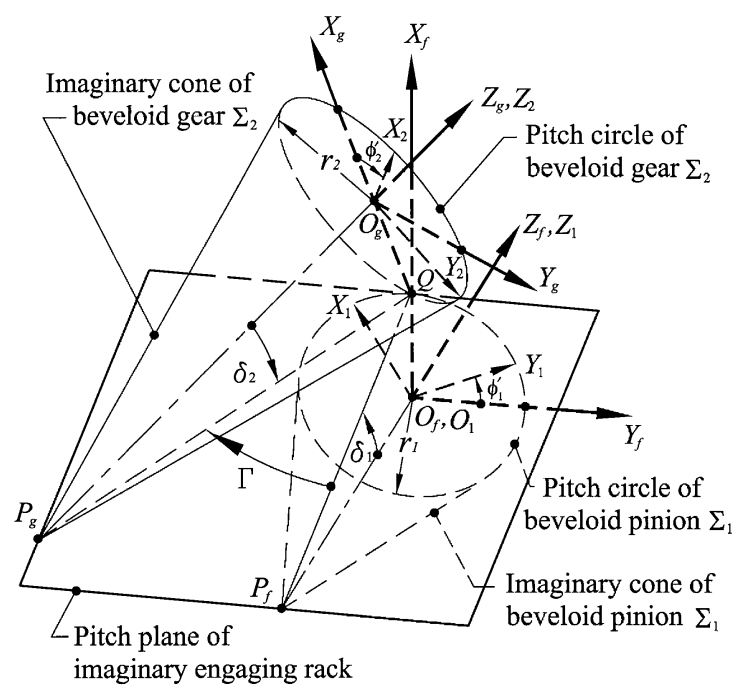

Fig. 4. Schematic relationship for the meshing of pinion, gear and the pitch plane of the imaginary engaging rack. 
their respective pitch radii, and $P_{f}$ and $P_{g}$ are the apexes of the imaginary cones; $\overline{P_{f} O_{f}}$ and $\overline{P_{g} O_{g}}$ are the axes of the imaginary cones. The pitch circles of the pinion and gear are in tangency at point $Q$. In addition, the tangent lines of the two imaginary cones with respect to the pitch plane of the imaginary engaging rack, $\overline{P_{f} Q}$ and $\overline{P_{g} Q}$, form an angle $\Gamma=\beta_{F}+\beta_{G}$ (or $\Gamma=\beta_{F}+\beta_{G}+\pi$ ). Herein, $\beta_{F}$ and $\beta_{G}$ denote the helix angle on the pitch plane of the rack cutter in the generation of the pinion $\Sigma_{1}$ and the gear $\Sigma_{2}$, respectively. The preceding meshing model of the beveloid gear pair is designed to simulate the meshing of a beveloid gear pair with crossed axes. Consider that when the angle $\Gamma=0$ or $\Gamma=\pi$, the axes of two imaginary cones intersect and form an intersected angle of $\delta_{1}+\delta_{2}$ (when $\Gamma=0$ ) or $\left|\delta_{1}-\delta_{2}\right|$ (when $\Gamma=\pi$ ). Thus, the meshing model regresses to the case which can simulate the meshing of beveloid gears with intersected axes. Furthermore, when the intersected angle becomes zero, the axes of the two imaginary cones become parallel, and the meshing model regresses again to the case which can simulate the meshing of beveloid gears with parallel axes.

To investigate the meshing of beveloid gear pair with assembly errors, auxiliary coordinate systems $S_{e}\left(X_{e}, Y_{e}, Z_{e}\right), S_{h}\left(X_{h}, Y_{h}, Z_{h}\right)$ and $S_{v}\left(X_{v}, Y_{v}, Z_{v}\right)$ have been set up to simulate the assembly errors of the gear $\Sigma_{2}$ as depicted in Fig. 5. Coordinate system $S_{e}$ is set up and keeps its orientation with respect to coordinate system $S_{g}$. However, the origin $O_{e}$ of coordinate system $S_{e}$ has an offset deviation from the origin $O_{g}$ of coordinate system $S_{g}$. The offset $\overline{O_{g} O_{e}}=\Delta \boldsymbol{d}=\left(\Delta x_{g}, \Delta y_{g}, \Delta z_{g}\right)$ indicates the mounting position deviation of the gear $\Sigma_{2}$. Moreover, coordinate system $S_{h}$ simulates the gear $\Sigma_{2}$ having a horizontal misaligned angle $\Delta \gamma_{h}$ with respect to coordinate system $S_{e}$, while coordinate system $S_{v}$ simulates the gear $\Sigma_{2}$ having a vertical misaligned angle $\Delta \gamma_{v}$ with respect to coordinate system $S_{h}$. Hence, coordinate system $S_{v}$ is the reference coordinate system for the gear coordinate system $S_{2}$ when assembly errors $\Delta \boldsymbol{d}, \Delta \gamma_{h}$ and $\Delta \gamma_{v}$ exist.

Applying the coordinate transformation matrix equation, the position vectors and unit normal vectors of the pinion $\Sigma_{1}$ and the gear $\Sigma_{2}$ can be represented in the coordinate system $S_{f}$. The mating pinion and gear must satisfy the following conditions at their instantaneous contact point $[5,6]$ :

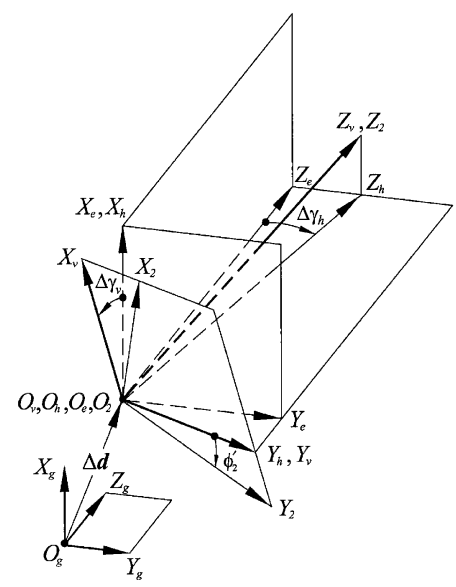

Fig. 5. Assembly errors of the beveloid gear. 


$$
\boldsymbol{R}_{f}^{(1)}=\boldsymbol{R}_{f}^{(2)}
$$

and

$$
\boldsymbol{n}_{f}^{(1)}= \pm \boldsymbol{n}_{f}^{(2)},
$$

where $\boldsymbol{R}_{f}^{(1)}$ and $\boldsymbol{R}_{f}^{(2)}$ are the position vectors while $\boldsymbol{n}_{f}^{(1)}$ and $\boldsymbol{n}_{f}^{(2)}$ denote the unit normal vectors of pinion $\Sigma_{1}$ and gear $\Sigma_{2}$, represented in coordinate system $S_{f}$, respectively. In a three-dimensional space, Eqs. (9) and (10) form a system of five independent non-linear equations with six unknowns $\phi_{1}^{\prime}, \ell_{F}, u_{F}, \phi_{2}^{\prime}, \ell_{G}$ and $u_{G}$. By choosing the pinion rotational angle $\phi_{1}^{\prime}$ as an input variable, all other unknowns can be solved in terms of $\phi_{1}^{\prime}$. The instantaneous contact points on the pinion and gear surfaces can be obtained by substituting the solved unknowns into the pinion and gear tooth-surface equations. The deviation of the real gear rotational angle $\phi_{2}^{\prime}\left(\phi_{1}^{\prime}\right)$ from its ideal rotational angle is defined as the transmission error (TE) which can be expressed as follows:

$$
\mathrm{TE}=\phi_{2}^{\prime}\left(\phi_{1}^{\prime}\right)-\phi_{1}^{\prime} \frac{N_{1}}{N_{2}},
$$

where $N_{1}$ and $N_{2}$ denote the number of teeth of the pinion and gear, respectively.

\section{Curvature analysis and contact ellipses}

In this study, the methodologies proposed by Litvin $[5,6]$ evaluating the principal directions and curvatures of the generated surfaces in terms of their corresponding generating tool surfaces are adopted to obtain the contact ellipses of the mating beveloid gear pairs with nonparallel axes. Notably, only the derivation processes of curvature relationships between the cutter surface $\Sigma_{F}$ and the generated pinion surfaces $\Sigma_{1}$ are shown hereinafter. The principal directions and curvatures of the gear surfaces $\Sigma_{2}$ can also be derived as well by the same procedure.

\subsection{Principal directions and curvatures of rack cutter surface $\Sigma_{F}$}

The position vector and unit normal vector of the cutter surface $\Sigma_{F}$ are expressed in Eqs. (2) and (5), respectively. According to Rodrigues' equation, the principal curvatures of the cutter surface $\Sigma_{F}$ can be obtained as $[5,6]$

$$
\kappa_{\mathrm{I}, \mathrm{II}}^{(F)} \boldsymbol{V}_{\mathrm{r}}^{(F)}=-\dot{\boldsymbol{n}}_{r}^{(F)},
$$

where $\boldsymbol{V}_{\mathrm{r}}^{(F)}$ is the relative velocity at the contact point in its motion over the cutter surface $\Sigma_{F} ; \dot{\boldsymbol{n}}_{r}^{(F)}$ is the velocity of the tip of the surface unit normal in the above motion; and $\kappa_{\mathrm{I}, \mathrm{II}}^{(F)}$ represents the two principal curvatures of the surface at the contact point. Since $u_{F}$ and $\ell_{F}$ are the surface parameters of the rack cutter surface $\Sigma_{F}$, Eqs. (2), (5) and (12) are used to calculate the principal directions and curvatures of the rack cutter surface $\Sigma_{F}$ as follows: 
1. When $\left(\mathrm{d} \ell_{F} / \mathrm{d} t\right)=0$, the first principal direction $\boldsymbol{i}_{\mathrm{I}}^{(F)}$ and curvature $\kappa_{\mathrm{I}}^{(F)}$ are derived as follows:

$$
\boldsymbol{i}_{\mathrm{I}}^{(F)}=\frac{\boldsymbol{V}_{\mathrm{rI}}^{(F)}}{\left|\boldsymbol{V}_{\mathrm{rI}}^{(F)}\right|}=\left[\begin{array}{c}
\sin \delta_{1} \cos \beta_{F} \\
\sin \beta_{F} \\
\cos \delta_{1} \cos \beta_{F}
\end{array}\right], \quad \text { and } \quad \kappa_{\mathrm{I}}^{(F)}=0 .
$$

2. When $\left(\mathrm{d} u_{F} / \mathrm{d} t\right)=0$, the secondary principal direction $\boldsymbol{i}_{\mathrm{II}}^{(F)}$ and curvature $\kappa_{\mathrm{II}}^{(F)}$ are derived as follows:

$$
\boldsymbol{i}_{\mathrm{II}}^{(F)}=\frac{\boldsymbol{V}_{\mathrm{rII}}^{(F)}}{\left|\boldsymbol{V}_{\mathrm{rII}}^{(F)}\right|}=\left[\begin{array}{c}
\cos \delta_{1} \cos \alpha_{n}^{(F)}-\sin \delta_{1} \sin \beta_{F} \sin \alpha_{n}^{(F)} \\
\cos \beta_{F} \sin \alpha_{n}^{(F)} \\
-\sin \delta_{1} \cos \alpha_{n}^{(F)}-\cos \delta_{1} \sin \beta_{F} \sin \alpha_{n}^{(F)}
\end{array}\right], \quad \text { and } \quad \kappa_{\mathrm{II}}^{(F)}=0 .
$$

Herein, unit vectors $\boldsymbol{i}_{\mathrm{I}}^{(F)}$ and $\boldsymbol{i}_{\mathrm{II}}^{(F)}$ are represented in the coordinate system $S_{b}^{(F)}\left(X_{b}^{(F)}, Y_{b}^{(F)}, Z_{b}^{(F)}\right)$ as shown in Fig. 3. Noted that the principal curvatures $\kappa_{\mathrm{I}}^{(F)}$ and $\kappa_{\mathrm{II}}^{(F)}$ are both zero since the generating surface $\Sigma_{F}$ is a plane.

\subsection{Principal directions and curvatures of the generated pinion tooth surface $\Sigma_{l}$}

The generated pinion tooth surface $\Sigma_{1}$ and the cutter surface $\Sigma_{F}$ are in line contact and in continuous tangency at every instant during the generation process. Therefore, the principal directions and curvatures of the generated pinion tooth surface $\Sigma_{1}$ can be determined by applying the following equations $[5,6]$ :

$$
\begin{aligned}
& \tan 2 \sigma^{(F 1)}=\frac{2 F^{(1)}}{\kappa_{\mathrm{I}}^{(F)}-\kappa_{\mathrm{II}}^{(F)}+G^{(1)}}, \\
& \kappa_{\mathrm{I}}^{(1)}+\kappa_{\mathrm{II}}^{(1)}=\kappa_{\mathrm{I}}^{(F)}+\kappa_{\mathrm{II}}^{(F)}+S^{(1)},
\end{aligned}
$$

and

$$
\kappa_{\mathrm{I}}^{(1)}-\kappa_{\mathrm{II}}^{(1)}=\frac{\kappa_{\mathrm{I}}^{(F)}-\kappa_{\mathrm{II}}^{(F)}+G^{(1)}}{\cos 2 \sigma^{(F 1)}},
$$

where

$$
\begin{aligned}
& F^{(1)}=\frac{a_{31}^{(1)} a_{32}^{(1)}}{b_{3}^{(1)}+\left(\boldsymbol{V}^{(F 1)} \cdot \boldsymbol{i}_{\mathrm{I}}^{(F)}\right) a_{31}^{(1)}+\left(\boldsymbol{V}^{(F 1)} \cdot \boldsymbol{i}_{\mathrm{II}}^{(F)}\right) a_{32}^{(1)}}, \\
& G^{(1)}=\frac{\left(a_{31}^{(1)}\right)^{2}-\left(a_{32}^{(1)}\right)^{2}}{b_{3}^{(1)}+\left(\boldsymbol{V}^{(F 1)} \cdot \boldsymbol{i}_{\mathrm{I}}^{(F)}\right) a_{31}^{(1)}+\left(\boldsymbol{V}^{(F 1)} \cdot \boldsymbol{i}_{\mathrm{II}}^{(F)}\right) a_{32}^{(1)}}, \\
& S^{(1)}=\frac{\left(a_{31}^{(1)}\right)^{2}+\left(a_{32}^{(1)}\right)^{2}}{b_{3}^{(1)}+\left(\boldsymbol{V}^{(F 1)} \cdot \boldsymbol{i}_{\mathrm{I}}^{(F)}\right) a_{31}^{(1)}+\left(\boldsymbol{V}^{(F 1)} \cdot \boldsymbol{i}_{\mathrm{II}}^{(F)}\right) a_{32}^{(1)}}, \\
& a_{31}^{(1)}=\left[\boldsymbol{n}^{(F)} \boldsymbol{\omega}^{(F 1)} \boldsymbol{i}_{\mathrm{I}}^{(F)}\right]-\kappa_{\mathrm{I}}^{(F)}\left(\boldsymbol{V}^{(F 1)} \cdot \boldsymbol{i}_{\mathrm{I}}^{(F)}\right),
\end{aligned}
$$




$$
a_{32}^{(1)}=\left[\boldsymbol{n}^{(F)} \boldsymbol{\omega}^{(F 1)} \boldsymbol{i}_{\mathrm{II}}^{(F)}\right]-\kappa_{\mathrm{II}}^{(F)}\left(\boldsymbol{V}^{(F 1)} \cdot \boldsymbol{i}_{\mathrm{II}}^{(F)}\right)
$$

and

$$
b_{3}^{(1)}=\left[\boldsymbol{n}^{(F)} \boldsymbol{\omega}^{(1)} \boldsymbol{V}_{\mathrm{tr}}^{(F)}\right]-\left[\boldsymbol{n}^{(F)} \boldsymbol{\omega}^{(F)} \boldsymbol{V}_{\mathrm{tr}}^{(1)}\right] .
$$

All the vectors in Eqs. (15)-(23) are represented in the coordinate system $S_{b}^{(F)}$. Herein, $\boldsymbol{i}_{\mathrm{I}}^{(1)}$ and $\boldsymbol{i}_{\mathrm{II}}^{(1)}$ are the first and second principal directions of the generated pinion tooth surface $\Sigma_{1} ; \kappa_{\mathrm{I}}^{(1)}$ and $\kappa_{\mathrm{II}}^{(1)}$ represent the first and second principal curvatures of $\Sigma_{1}$; and $\sigma^{(F 1)}$ is the angle formed by the first principal directions of the cutter surface $\boldsymbol{i}_{\mathrm{I}}^{(F)}$ and the generated pinion surface $\boldsymbol{i}_{\mathrm{I}}^{(1)}$. It is recalled that the vector $\omega^{(F)}=0$ due to the translational motion of rack cutter $\Sigma_{F}$. Therefore, the relative angular velocity between the rack cutter surface $\Sigma_{F}$ and the generated pinion tooth surface $\Sigma_{1}$, can be expressed as

$$
\omega^{(F 1)}=\omega^{(F)}-\omega^{(1)}=-\omega^{(1)}=\left[\begin{array}{c}
0 \\
0 \\
\omega_{1}
\end{array}\right] .
$$

Meanwhile, the transfer velocity of the rack cutter $\Sigma_{F}$ is

$$
\boldsymbol{V}_{\mathrm{tr}}^{(F)}=\left[\begin{array}{c}
0 \\
-\omega_{1} r_{1} \\
0
\end{array}\right]
$$

In addition, the position vector of the contact point on the generated pinion tooth surface $\Sigma_{1}$, represented in coordinate system $S_{b}^{(F)}$, can be expressed as

$$
\boldsymbol{R}_{b}^{(1)}=\left[\begin{array}{c}
x_{c}^{(F)}+r_{1} \\
y_{c}^{(F)}-r_{1} \phi_{1} \\
z_{c}^{(F)}
\end{array}\right]
$$

where $x_{c}^{(F)}, y_{c}^{(F)}$ and $z_{c}^{(F)}$ are expressed in Eq. (2). Therefore, the transfer velocity of contact point on $\Sigma_{1}$ is

$$
\boldsymbol{V}_{\mathrm{tr}}^{(1)}=\omega^{(1)} \times \boldsymbol{R}_{b}^{(1)}=\omega_{1}\left[\begin{array}{c}
y_{c}^{(F)}-r_{1} \phi_{1} \\
-x_{c}^{(F)}-r_{1} \\
0
\end{array}\right] .
$$

Thus, the relative velocity between surfaces $\Sigma_{F}$ and $\Sigma_{1}$, represented in coordinate system $S_{b}^{(F)}$, can be obtained as

$$
\boldsymbol{V}^{(F 1)}=\boldsymbol{V}_{\mathrm{tr}}^{(F)}-\boldsymbol{V}_{\mathrm{tr}}^{(1)}=\omega_{1}\left[\begin{array}{c}
-y_{c}^{(F)}+r_{1} \phi_{1} \\
x_{c}^{(F)} \\
0
\end{array}\right] .
$$

In addition, two common terms appeared in Eqs. (18)-(20) can be obtained by applying Eqs. (13), (14) and (28):

$$
\boldsymbol{V}^{(F 1)} \cdot \boldsymbol{i}_{\mathrm{I}}^{(F)}=\omega_{1}\left[\left(-y_{c}^{(F)}+r_{1} \phi_{1}\right) \sin \delta_{1} \cos \beta_{F}+x_{c}^{(F)} \sin \beta_{F}\right],
$$


and

$$
\begin{aligned}
\boldsymbol{V}^{(F 1)} \cdot \boldsymbol{i}_{\mathrm{II}}^{(F)}= & \omega_{1}\left[\left(-y_{c}^{(F)}+r_{1} \phi_{1}\right)\left(\cos \delta_{1} \cos \alpha_{n}^{(F)}-\sin \delta_{1} \sin \beta_{F} \sin \alpha_{n}^{(F)}\right)\right. \\
& \left.+x_{c}^{(F)}\left(\cos \beta_{F} \sin \alpha_{n}^{(F)}\right)\right] .
\end{aligned}
$$

Furthermore, since $\kappa_{\mathrm{I}}^{(F)}=\kappa_{\mathrm{II}}^{(F)}=0$ and $\omega^{(F)}=0$, coefficients $a_{31}^{(1)}, a_{32}^{(1)}$ and $b_{3}^{(1)}$ can be attained as follows:

$$
\begin{aligned}
& a_{31}^{(1)}=\left[n^{(F)} \boldsymbol{\omega}^{(F 1)} \boldsymbol{i}_{\mathrm{I}}^{(F)}\right]=\omega_{1} \sin \delta_{1} \cos \alpha_{n}^{(F)}+\omega_{1} \cos \delta_{1} \sin \beta_{F} \sin \alpha_{n}^{(F)}, \\
& a_{32}^{(1)}=\left[\boldsymbol{n}^{(F)} \boldsymbol{\omega}^{(F 1)} \boldsymbol{i}_{\mathrm{II}}^{(F)}\right]=\omega_{1} \cos \delta_{1} \cos \beta_{F},
\end{aligned}
$$

and

$$
b_{3}^{(1)}=\left[\boldsymbol{n}^{(F)} \boldsymbol{\omega}^{(1)} \boldsymbol{V}_{\mathrm{tr}}^{(F)}\right]=r_{1} \omega_{1}^{2}\left(\sin \delta_{1} \sin \beta_{F} \cos \alpha_{n}^{(F)}+\cos \delta_{1} \sin \alpha_{n}^{(F)}\right) .
$$

It is obvious that all terms needed to express $F^{(1)}, G^{(1)}$ and $S^{(1)}$ have been derived completely. Consequently, Eqs. (15)-(17) determine the principal curvatures and directions of the pinion tooth surface $\Sigma_{1}$ represented in coordinate system $S_{b}^{(F)}$. Notably, the principal directions of $\Sigma_{1}$ can be represented in coordinate system $S_{1}$ by applying the coordinate transformation matrix equation. Similarly, the principal curvatures and directions of any point on the gear tooth surface $\Sigma_{2}$ generated by cutter $\Sigma_{G}$ can be derived as well and expressed in coordinate system $S_{2}$ by the same procedure.

\subsection{Contact ellipses}

According to the TCA results, the principal directions $\boldsymbol{i}_{\mathrm{I}}^{(1)}$ and $\boldsymbol{i}_{\mathrm{I}}^{(2)}$ represented in coordinate systems $S_{1}$ and $S_{2}$, respectively, can be described in coordinate system $S_{f}$ by applying the coordinate transformation matrix equation. Obviously, at any instantaneous contact point, $\boldsymbol{i}_{\mathrm{I}}^{(1)}$ and $\boldsymbol{i}_{\mathrm{I}}^{(2)}$ are located on the common tangent plane of the mating surfaces $\Sigma_{1}$ and $\Sigma_{2}$, as shown in Fig. 6 . The orientation of the contact ellipse is determined by the angle $\gamma$ which can be represented with the following equations $[5,6]$ :

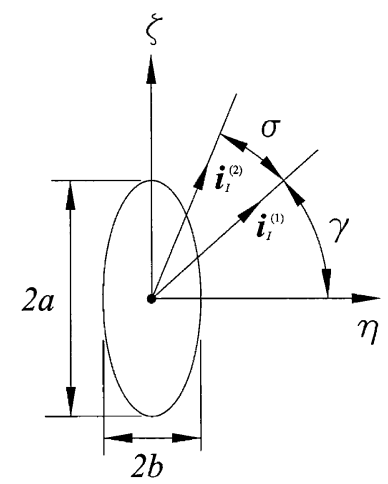

Fig. 6. Orientation and dimensions of contact ellipse. 


$$
\tan 2 \gamma=\frac{g_{2} \sin 2 \sigma}{g_{1}-g_{2} \cos 2 \sigma},
$$

where

$$
g_{1}=\kappa_{\mathrm{I}}^{(1)}-\kappa_{\mathrm{II}}^{(1)}
$$

and

$$
g_{2}=\kappa_{\mathrm{I}}^{(2)}-\kappa_{\mathrm{II}}^{(2)} .
$$

Meanwhile, angle $\sigma$ is formed by the first principal directions of the gear and pinion tooth surfaces $\boldsymbol{i}_{\mathrm{I}}^{(2)}$ and $\boldsymbol{i}_{\mathrm{I}}^{(1)}$, and it can be evaluated by

$$
\sigma=\cos ^{-1}\left(\boldsymbol{i}_{\mathrm{I}}^{(1)} \cdot \boldsymbol{i}_{\mathrm{I}}^{(2)}\right) \text {. }
$$

The half length of the major and minor axes of the contact ellipse, $a$ and $b$, can be expressed in terms of the elastic approach $\Delta$ by $[5,6]$

$$
a=\left|\frac{\Delta}{A}\right|^{\frac{1}{2}},
$$

and

$$
b=\left|\frac{\Delta}{B}\right|^{\frac{1}{2}},
$$

where

$$
\begin{aligned}
& A=\frac{1}{4}\left[\kappa_{\Sigma}^{(1)}-\kappa_{\Sigma}^{(2)}-\left(g_{1}^{2}-2 g_{1} g_{2} \cos 2 \sigma+g_{2}^{2}\right)^{1 / 2}\right], \\
& B=\frac{1}{4}\left[\kappa_{\Sigma}^{(1)}-\kappa_{\Sigma}^{(2)}+\left(g_{1}^{2}-2 g_{1} g_{2} \cos 2 \sigma+g_{2}^{2}\right)^{1 / 2}\right], \\
& \kappa_{\Sigma}^{(1)}=\kappa_{\mathrm{I}}^{(1)}+\kappa_{\mathrm{II}}^{(1)},
\end{aligned}
$$

and

$$
\kappa_{\Sigma}^{(2)}=\kappa_{\mathrm{I}}^{(2)}+\kappa_{\mathrm{II}}^{(2)} .
$$

Thus, the orientation and dimension of the contact ellipse can be determined by utilizing Eqs. (34)-(39).

\section{Numerical illustrative examples for gear meshing simulations}

Fig. 7 illustrates three typical types of gear mounting for beveloid gear pairs with intersected, crossed and parallel axes. Applying the developed computer simulation programs, the TCA results can be obtained and the contact ellipses can be plotted on the contact tooth surfaces. The elastic approach $\Delta$ for the contact ellipse simulation is chosen as $0.00635 \mathrm{~mm}$, identical with the 


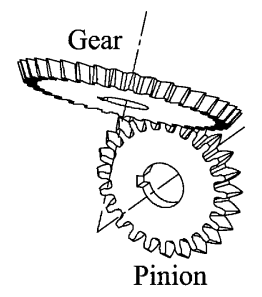

(a)

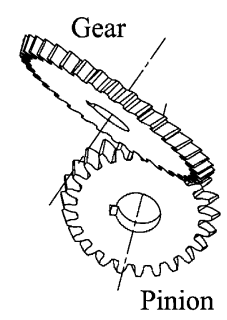

(b)

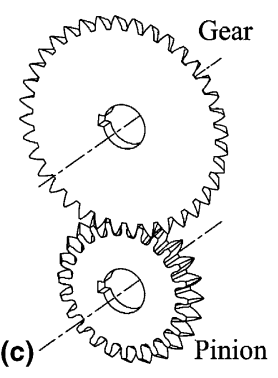

Fig. 7. Three typical mounting types of beveloid gear pairs: (a) intersected axes; (b) crossed axes; (c) parallel axes.

Table 1

Major design parameters of the beveloid pinion and gear

\begin{tabular}{lll}
\hline & Pinion & Gear \\
\hline Number of teeth & $N_{1}=24$ & $N_{2}=36$ \\
Face width (thickness of gear blank) & $F_{1}=14 \mathrm{~mm}$ & $F_{2}=14 \mathrm{~mm}$ \\
Normal pressure angle & $\alpha_{n}^{(F)}=20^{\circ}$ & $\alpha_{n}^{(G)}=20^{\circ}$ \\
Normal module & \multicolumn{2}{c}{$m_{n}=5(\mathrm{~mm} / \mathrm{teeth})$} \\
\hline
\end{tabular}

thickness of the coating paint used for contact pattern tests. Table 1 lists some major design parameters of the beveloid gear pair chosen for the following examples.

\subsection{Example 1: Straight beveloid gear pair with intersected axes}

In this example, the gear pair is composed of straight beveloid pinion and gear $\left(\beta_{F}=\beta_{G}=0^{\circ}\right)$ with cone angles $\delta_{1}=\delta_{2}=30^{\circ}$, mounted with an intersected angle of $60^{\circ}$, as Fig. 7(a) illustrates. Cases 1-3 simulate the meshing of the gear pair under the following assembly conditions:

Case 1: $\Delta \gamma_{h}=\Delta \gamma_{v}=0^{\circ}$ and $\Delta x_{g}=\Delta y_{g}=\Delta z_{g}=0 \mathrm{~mm}$ (ideal assembly condition).

Case 2: $\Delta \gamma_{h}=\Delta \gamma_{v}=0^{\circ}$ and $\Delta x_{g}=\Delta y_{g}=\Delta z_{g}=0.3 \mathrm{~mm}$.

Case 3: $\Delta \gamma_{h}=0.5^{\circ}, \Delta \gamma_{v}=-0.5^{\circ}$ and $\Delta x_{g}=\Delta y_{g}=\Delta z_{g}=0.3 \mathrm{~mm}$.

Case 1 is the ideal assembly condition. Case 2 indicates that the gear has a mounting position deviation but there is no axial misalignment. Case 3 indicates that the gear pair has both mounting position deviation and axial misalignments. The bearing contact of a straight beveloid gear pair with intersected axes is a point contact. The TCA results and the TE were calculated and summarized in Table 2. Meanwhile, the loci of contact points and their corresponding contact ellipses on the pinion surface were illustrated in Fig. 8. Even when the gear pair meshes under various assembly errors, the TEs remain zero and the loci of contact points remain in the middle region of the tooth flank. These results indicate that the straight beveloid gear pair mounted with intersected axes is insensitive to assembly errors. Under some circumstances, intersected straight beveloid gears can replace bevel gears and take the advantage of insensitivity to the gear assembly errors. However, the contact ellipses of a beveloid gear pair with intersected axes are relatively small, especially with large cone angles. Therefore, tooth surface durability is generally low owing to its high contact stress. Considering the straight beveloid gear pair in this example, one curve 
Table 2

TCA results of the straight beveloid gear pair with intersected axes

\begin{tabular}{llllllll}
\hline Case & $\phi_{1}^{\prime}($ deg. $)$ & $\ell_{F}$ & $u_{F}$ & $\phi_{2}^{\prime}($ deg. $)$ & \multicolumn{1}{l}{$\ell_{G}$} & $u_{G}$ & TE (arc-sec.) \\
\hline 1 & 6.0 & 8.813 & 0.000 & 189.000 & 1.829 & 0.000 & 0.000 \\
& 3.0 & 7.738 & 0.000 & 187.000 & 2.903 & 0.000 & 0.000 \\
& 0.0 & 6.664 & 0.000 & 185.000 & 3.978 & 0.000 & 0.000 \\
& -3.0 & 5.590 & 0.000 & 183.000 & 5.052 & 0.000 & 0.000 \\
& -6.0 & 4.515 & 0.000 & 181.000 & 6.127 & 0.000 & 0.000 \\
2 & 6.0 & 9.027 & -0.395 & 189.096 & 2.051 & -0.504 & 0.000 \\
& 3.0 & 7.953 & -0.395 & 187.096 & 3.125 & -0.504 & 0.000 \\
& 0.0 & 6.878 & -0.395 & 185.096 & 4.200 & -0.504 & 0.000 \\
& -3.0 & 5.804 & -0.395 & 183.096 & 5.274 & -0.504 & 0.000 \\
& -6.0 & 4.729 & -0.395 & 181.096 & 6.349 & -0.504 & 0.000 \\
& 6.0 & 9.033 & 0.099 & 189.185 & 1.620 & 0.669 & 0.000 \\
& 3.0 & 7.958 & 0.099 & 187.185 & 2.695 & 0.669 & 0.000 \\
& 0.0 & 6.884 & 0.099 & 185.185 & 3.769 & 0.669 & 0.000 \\
& -3.0 & 5.809 & 0.099 & 183.185 & 4.844 & 0.669 & 0.000 \\
& -6.0 & 4.735 & 0.099 & 181.185 & 5.918 & 0.669 & 0.000 \\
\hline
\end{tabular}

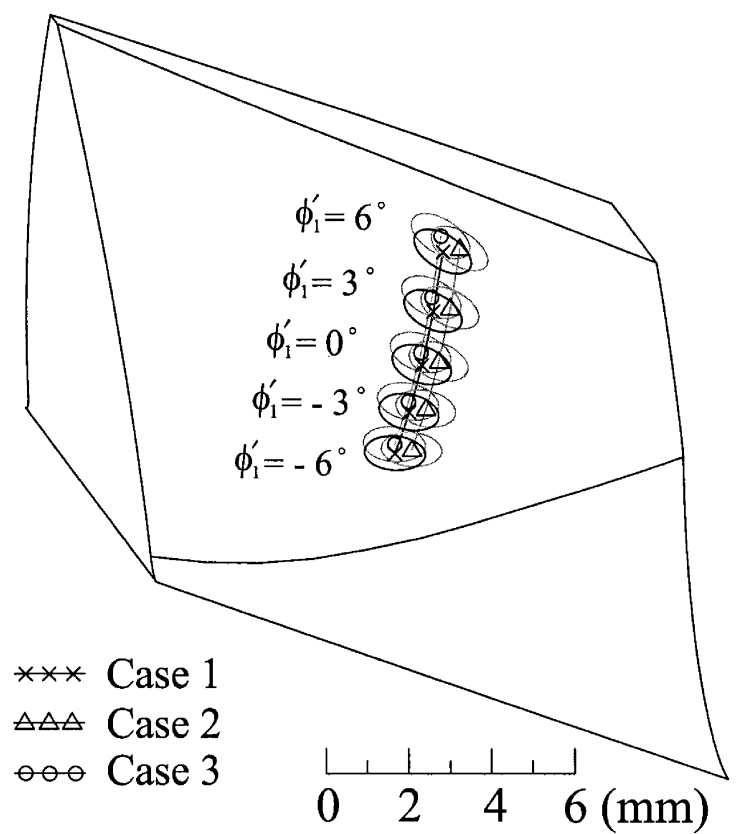

Fig. 8. Bearing contact of a straight beveloid gear pair with intersected axes.

$\left(\beta_{F}=\beta_{G}=0^{\circ}\right)$ in Fig. 9 shows how with the increase of cone angles $\delta_{1}$ and $\delta_{2}$, the ratio between the major and minor axes of the contact ellipse, $a / b$, decrease significantly. Notably, the ratio $a / b$ approaches to infinity when the cone angles $\delta_{1}$ and $\delta_{2}$ tend to zero, which represents a spur gear pair in line contact. 


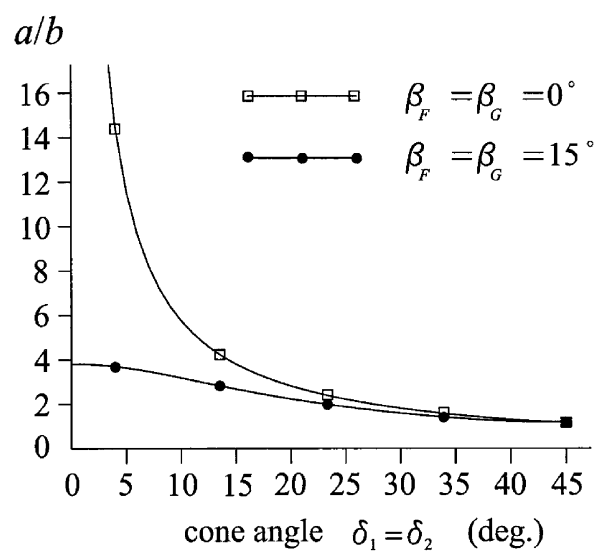

Fig. 9. The ratio $a / b$ with respect to the cone angle.

\subsection{Example 2: Helical beveloid gear pair with crossed axes}

Fig. 7(b) illustrates a beveloid gear pair consisting of helical beveloid pinion and gear with crossed axes. The cone angles of the helical beveloid pinion and gear are $\delta_{1}=\delta_{2}=20^{\circ}$, and the helix angles on the pitch planes of the rack cutters for the pinion and gear are $\beta_{F}=\beta_{G}=15^{\circ}$ (right handed). The crossed angle formed by axes $Z_{f}$ and $Z_{g}$, as shown in Fig. 4 , is calculated as $49.6284^{\circ}$ by applying the algorithms proposed by Mitome [2]. This example investigates the meshing simulations of gear pairs under the following assembly conditions:

Case 4: $\Delta \gamma_{h}=\Delta \gamma_{v}=0^{\circ}$ and $\Delta x_{g}=\Delta y_{g}=\Delta z_{g}=0 \mathrm{~mm}$ (ideal assembly condition).

Case 5: $\Delta \gamma_{h}=\Delta \gamma_{v}=0^{\circ}$ and $\Delta x_{g}=\Delta y_{g}=\Delta z_{g}=0.3 \mathrm{~mm}$.

Case 6: $\Delta \gamma_{h}=0.5^{\circ}, \Delta \gamma_{v}=-0.5^{\circ}$ and $\Delta x_{g}=\Delta y_{g}=\Delta z_{g}=0.3 \mathrm{~mm}$.

Table 3 summarizes the TCA results and TEs of the gear pair, and Fig. 10 illustrates the loci of contact points and their corresponding contact ellipses on the pinion surface. Even under various assembly errors, the TEs remain zero and the loci of contact points also remain in the middle region of the tooth flank. Therefore, the helical beveloid gear pair with crossed axes is also insensitive to assembly errors. Another curve in Fig. 9 indicates the relationship between the cone angles and the ratio $a / b$ of this helical beveloid gear pair with $\beta_{F}=\beta_{G}=15^{\circ}$. It is reasonable to find the ratio $a / b$ approaches to a limited value when the cone angles tend to zero, which represents that a crossed axes helical gear pair is in point contact.

\subsection{Example 3: Straight beveloid gear pair with parallel axes}

Fig. 7(c) illustrates a straight beveloid gear pair mounted with the parallel axes. The pinion and gear are identical to those in Example 1 (cf. Section 5.1). However, the gear is now turned over to allow the heel of the gear to engage with the toe of the pinion. Thus, the axes of pinion and gear are now parallel. Cases 7-9 simulate the meshing of the gear pair under the following assembly conditions: 
Table 3

TCA results of the helical beveloid gear pair with crossed axes

\begin{tabular}{|c|c|c|c|c|c|c|c|}
\hline Case & $\phi_{1}^{\prime}$ (deg.) & $\ell_{F}$ & $u_{F}$ & $\phi_{2}^{\prime}$ (deg.) & $\ell_{G}$ & $u_{G}$ & TE (arc-sec.) \\
\hline 4 & $\begin{array}{r}6.0 \\
3.0 \\
0.0 \\
-3.0 \\
-6.0\end{array}$ & $\begin{array}{l}8.813 \\
7.738 \\
6.664 \\
5.590 \\
4.515\end{array}$ & $\begin{array}{r}1.684 \\
0.842 \\
0.000 \\
-0.842 \\
-1.684\end{array}$ & $\begin{array}{l}189.000 \\
187.000 \\
185.000 \\
183.000 \\
181.000\end{array}$ & $\begin{array}{l}1.829 \\
2.903 \\
3.978 \\
5.052 \\
6.127\end{array}$ & $\begin{array}{l}-3.788 \\
-2.946 \\
-2.104 \\
-1.263 \\
-0.421\end{array}$ & $\begin{array}{l}0.000 \\
0.000 \\
0.000 \\
0.000 \\
0.000\end{array}$ \\
\hline 5 & $\begin{array}{r}6.0 \\
3.0 \\
0.0 \\
-3.0 \\
-6.0\end{array}$ & $\begin{array}{l}9.030 \\
7.955 \\
6.881 \\
5.806 \\
4.732\end{array}$ & $\begin{array}{r}1.197 \\
0.355 \\
-0.487 \\
-1.328 \\
-2.170\end{array}$ & $\begin{array}{l}189.125 \\
187.125 \\
185.125 \\
183.125 \\
181.125\end{array}$ & $\begin{array}{l}2.021 \\
3.096 \\
4.170 \\
5.245 \\
6.319\end{array}$ & $\begin{array}{l}-4.423 \\
-3.581 \\
-2.739 \\
-1.897 \\
-1.056\end{array}$ & $\begin{array}{l}0.000 \\
0.000 \\
0.000 \\
0.000 \\
0.000\end{array}$ \\
\hline 6 & $\begin{array}{r}6.0 \\
3.0 \\
0.0 \\
-3.0 \\
-6.0\end{array}$ & $\begin{array}{l}9.034 \\
7.960 \\
6.885 \\
5.811 \\
4.736\end{array}$ & $\begin{array}{r}2.158 \\
1.316 \\
0.474 \\
-0.368 \\
-1.209\end{array}$ & $\begin{array}{l}189.063 \\
187.063 \\
185.063 \\
183.063 \\
181.063\end{array}$ & $\begin{array}{l}1.710 \\
2.784 \\
3.859 \\
4.933 \\
6.008\end{array}$ & $\begin{array}{r}-2.698 \\
-1.856 \\
-1.014 \\
-0.172 \\
0.669\end{array}$ & $\begin{array}{l}0.000 \\
0.000 \\
0.000 \\
0.000 \\
0.000\end{array}$ \\
\hline
\end{tabular}

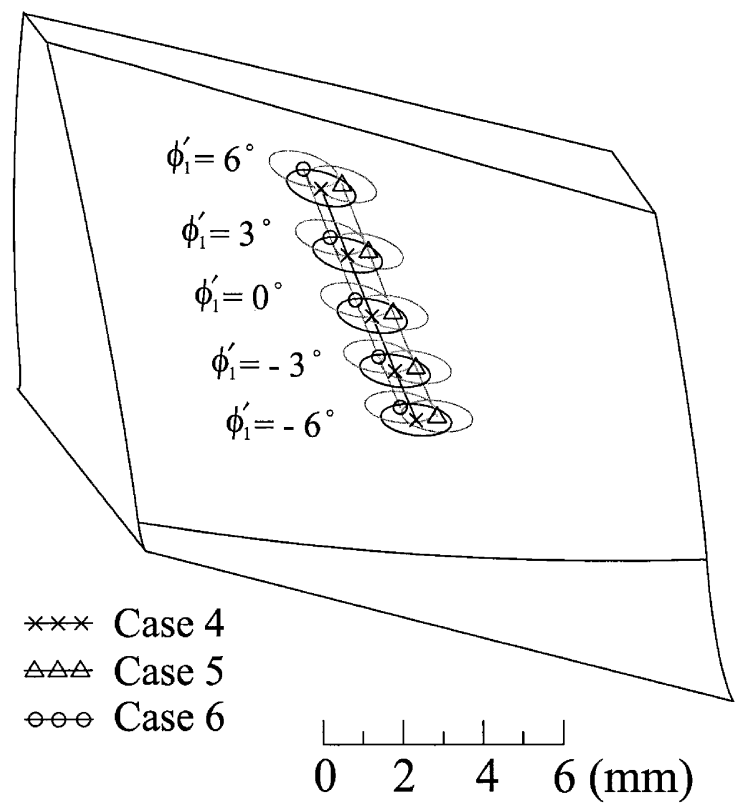

Fig. 10. Bearing contact of a helical beveloid gear pair with crossed axes.

Case 7: $\Delta \gamma_{h}=\Delta \gamma_{v}=0^{\circ}$ and $\Delta x_{g}=\Delta y_{g}=\Delta z_{g}=0 \mathrm{~mm}$ (ideal assembly condition).

Case 8: $\Delta \gamma_{h}=\Delta \gamma_{v}=0^{\circ}$ and $\Delta x_{g}=\Delta y_{g}=\Delta z_{g}=0.5 \mathrm{~mm}$.

Case 9: $\Delta \gamma_{h}=0.5^{\circ}, \Delta \gamma_{v}=0^{\circ}$ and $\Delta x_{g}=\Delta y_{g}=\Delta z_{g}=0.5 \mathrm{~mm}$ (edge contact condition). 


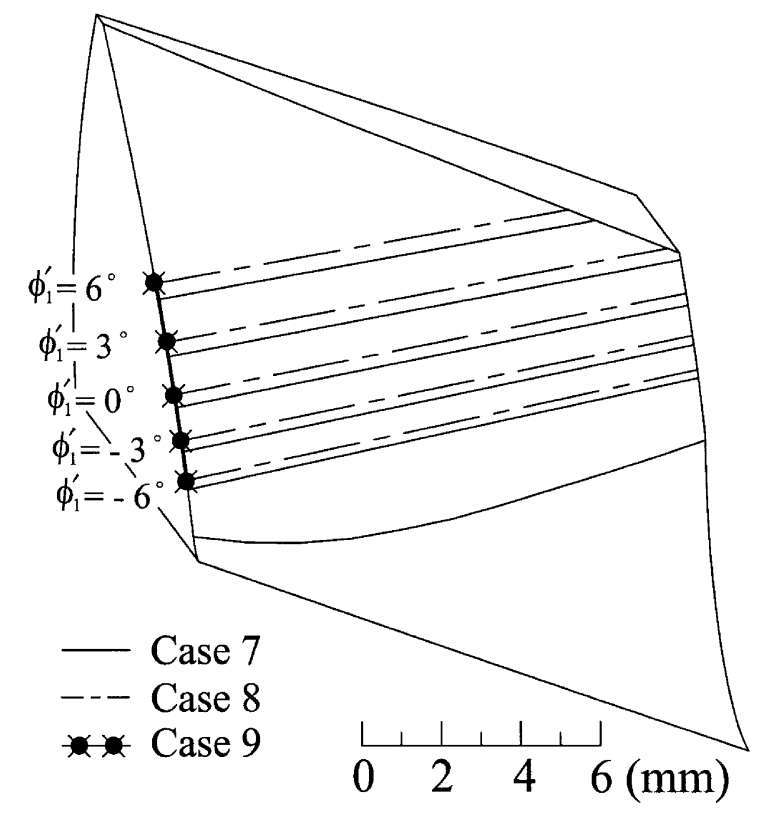

Fig. 11. Bearing contact of a straight beveloid gear pair with parallel axes.

Fig. 11 illustrates the bearing contacts of the gear pair on the pinion surface under three assembly conditions. Meanwhile, the TCA results and TEs are listed in Table 4. Under the ideal assembly condition (case 7) or with a mounting position deviation without an axial misalignment (case 8), similar to a spur or helical gear pair mounted with parallel axes, the bearing contact is a line contact and the TEs equal zero. However, when an axial misalignment occurs (case 9), the bearing contact becomes an edge contact and the TEs are induced as shown in Table 4. Notably, when the edge contact occurs, Eqs. (9) and (10) which describe the surface-to-surface continuous tangency are no longer applicable. Litvin [5,6] proposed the general concept of curve-to-surface continuous tangency to simulate edge contact in gear meshing. In Case 9, the edge contact occurs on the heel edge of the pinion which is in tangency with the surface of the gear. Therefore, the following equations must be observed:

$$
\begin{aligned}
& z_{1}=F_{1} / 2, \\
& \boldsymbol{R}_{f}^{(1)}=\boldsymbol{R}_{f}^{(2)},
\end{aligned}
$$

and

$$
\boldsymbol{t}_{f}^{(1)} \cdot \boldsymbol{n}_{f}^{(2)}=0
$$

where, $F_{1}$ is the face width of the pinion, and $z_{1}=F_{1} / 2$ represents the transverse plane on the heel side of the pinion. By combining $\boldsymbol{R}_{f}^{(1)}$ and Eq. (44), the curve which represents the edge on the heel of the beveloid pinion tooth is expressed in coordinate system $S_{f}$; in addition, the tangent of this edge can be derived and represented as $\boldsymbol{t}_{f}^{(1)}$. Eqs. (44)-(46) can be solved similarly to Eqs. (9) and (10). 
Table 4

TCA results of the straight beveloid gear pair with parallel axes

\begin{tabular}{lclllllc}
\hline Case & $\phi_{1}^{\prime}($ deg.) & $\ell_{F}$ & $u_{F}$ & $\phi_{2}^{\prime}($ deg.) & $\ell_{G}$ & $u_{G}$ & TE (arc-sec.) \\
\hline 7 & 6.0 & Line contact & & 189.000 & Line contact & & 0.000 \\
& 3.0 & & & 187.000 & & & 0.000 \\
& 0.0 & & & 185.000 & & & 0.000 \\
& -3.0 & & & 183.000 & & & 0.000 \\
& -6.0 & & 181.000 & & & 0.000 \\
8 & 6.0 & Line contact & & 189.273 & Line contact & & 0.000 \\
& 3.0 & & & 187.273 & & & 0.000 \\
& 0.0 & & & 185.273 & & & 0.000 \\
& -3.0 & & & 183.273 & & & 0.000 \\
& -6.0 & & & & & \\
& 6.0 & 4.852 & 7.828 & 189.320 & 5.932 & 7.064 & 20.213 \\
& 3.0 & 4.021 & 7.378 & 187.317 & 6.775 & 6.636 & 10.107 \\
& 0.0 & 3.190 & 6.927 & 185.314 & 7.618 & 6.207 & 0.000 \\
& -3.0 & 2.360 & 6.476 & 183.311 & 8.460 & 5.779 & -10.107 \\
& -6.0 & 1.529 & 6.026 & 181.308 & 9.303 & 5.350 & -20.213 \\
\hline
\end{tabular}

\section{Conclusions}

This study simulates the meshing of beveloid gear pairs with intersected, crossed and parallel axes. Bearing contact characteristics of the beveloid gear pairs under various assembly errors, and the contact ellipses of beveloid gear pairs with non-parallel axes are also investigated. Several illustrative numerical examples are presented as well. Based on the results attained in this study, we conclude the following:

1. Beveloid gear pairs with non-parallel axes exhibit point contact and are insensitive to assembly errors. Besides, the orientation and dimension of the contact ellipse can be determined. The results are helpful to further investigations on the contact characteristics and lubrication of this type of gear pairs.

2. The contact ellipses of non-parallel axes beveloid gear pairs are relatively small especially with large cone angles. Therefore, tooth surface durability is generally low owing to its high contact stress. Our upcoming research will provide some novel generation methods which can enlarge the contact ellipses of non-parallel axes beveloid gear pairs.

3. Straight beveloid gear pairs with parallel axes without axial misalignments can mesh conjugately in line contact. However, when axial misalignments are present, the edge contact replaces the line contact and unfavorable TEs, stress concentrations, noises and vibrations will occur. In real applications, one of the beveloid gears should be crowned to localize the bearing contact in the middle area of the tooth frank and to avoid edge contact.

\section{Acknowledgements}

The authors are grateful to the National Science Council of the ROC for the grant. Part of this work was performed under contract No. NSC 89-2212-E-009-013. 


\section{References}

[1] K. Mitome, Conical involute gear, Part 3: tooth action of a pair of gears, Bulletin of the JSME 28 (245) (1985) 27572764.

[2] K. Mitome, Conical involute gear (Design of nonintersecting-nonparallel-axis conical involute gear), JSME International Journal, Series III 34 (2) (1991) 265-270.

[3] K. Mitome, Infeed grinding of straight conical involute gear, JSME International Journal, Series C 36 (4) (1993) $537-542$.

[4] K. Mitome, Design of Miter conical involute gears based on tooth bearing, JSME International Journal, Series C 38 (2) (1995) 307-311.

[5] F.L. Litvin, Theory of Gearing, NASA Publication RP-1212, Washington DC, 1989.

[6] F.L. Litvin, Gear Geometry and Applied Theory, Prentice-Hall, Englewood Cliffs, NJ, 1994.

[7] H.E. Merritt, Gear, third ed., Issac Pitman and Sons, London, 1954.

[8] K. Mitome, Table sliding taper hobbing of conical gear using cylindrical hob, Part 1: theoretical analysis of table sliding taper hobbing, ASME Journal of Engineering for Industry 103 (1981) 446-451.

[9] K. Mitome, Inclining work-arbor taper hobbing of conical gear using cylindrical hob, ASME Journal of Mechanical Design 108 (1986) 135-141. 